\title{
ANALYSIS OF THE OPERATING COSTS OF PASSENGER CARS DECLARED BY VEHICLES' MANUFACTURERS AND OBTAINED BY USERS
}

\author{
Piotr Gorzelańczyk*, Bartlomiej Kelm \\ Stanislaw Staszic University of Applied Sciences in Pila, Pila, Poland \\ *E-mail of corresponding author: piotr.gorzelanczyk@puss.pila.pl
}

\begin{abstract}
Resume
Every year, there are more and more cars on the roads that cause pollution. To reduce it, conventional vehicles have started to be replaced by electric and hybrid vehicles. Therefore, the average fuel/energy consumption of electric, hybrid, spark ignition and self-ignition vehicles over a test distance of $100 \mathrm{~km}$ was investigated. The test results were then compared to the manufacturer's data and the average difference between the manufacturer's data and the test data is shown. The largest average difference in fuel consumption between the manufacturer's data and the test data was observed for hybrid vehicles (over $230 \%$ ) and the smallest for electric vehicles (less than $10 \%$ ) and spark ignition vehicles (almost $18 \%$ ). Considering costs, the largest difference between manufacturer's data and test data is observed, as in the previous case, in electric vehicles $(0.25 €)$ and the largest in hybrid vehicles (almost $6 €$ per 100 kilometers driven).
\end{abstract}

Available online: https://doi.org/10.26552/com.C.2022.2.A44-A52

\section{Article info}

Received 30 June 2021

Accepted 3 November 2021

Online 12 January 2022

\section{Keywords:}

car

fuel consumption

electricity

fuel costs

energy costs

ISSN 1335-4205 (print version)

ISSN 2585-7878 (online version)
One of the basic elements of human life is the ability to move. The constant development of technology has made it possible to cover short and long distances in a short time. The invention of the diesel engine contributed to the development of many areas of life, from daily commuting to industry and transcontinental transportation. Constant ocean travel, road traffic and road transport have left a carbon footprint for many years, which has exacerbated the problem of global warming. From the moment of the industrial revolution to the end of the 20th century, nobody thought about environmental protection or emission standards. The first passenger cars were not subject to any emissions restrictions. The emission problems were of interest to many researchers, for example [1-9]. For several years, an important element in those works on car concerns has been the project of replacing vehicles with an internal combustion engine with vehicles with an alternative drive. Shrinking oil resources, increasing environmental pollution and increasingly stringent exhaust emission standards gave rise to the idea of an electric and hybrid vehicle. Vehicles with alternative propulsion are to replace the conventional vehicles in order to contribute to the reduction of exhaust emissions. With the beginning of the new millennium, the stage of presenting ideas for modern electric and hybrid vehicles began and the development of electromobility in the world began. For this reason, the benefits for the user have been analyzed.
In order to comprehensively present what a motor vehicle is, one should refer to its history and cite the names that initiated a new stage in transport [10]. A motor vehicle is a complex device made of many interacting elements [11-13] and each of them plays an important role [14-16].

Internal combustion vehicles will be completely removed from the road by alternative propulsion vehicles over time [17]. Work on electric and hybrid vehicles began several dozen years ago [18-19].

One of the important aspects of the transition to alternative fuels is the environmental impact [20-21]. There are still newer limits and emission standards that contribute to the development of electric and hybrid vehicles. More and more such vehicles appear on the Polish and European market [22]. Research and statistical organizations are emerging on development of the electric and hybrid vehicle market [23]. Hybrid and electric vehicles have also been the subject of many research papers, for example [24-26].

Electricity is an indispensable component of hybrid and electric vehicles. For BEVs, it is the only fuel needed to drive a vehicle. Electricity is produced in Poland from fossil fuels and RES (Renewable Energy Sources) [27].

In 2020, the share of renewable energy in Poland was $17 \%$ (Figure 1), the rest being fossil fuels. Poland is at the forefront of statistics in terms of the consumption of fossil fuels for the production of electricity. One of 


\section{Energy mix for EU electricity generation, 2018}

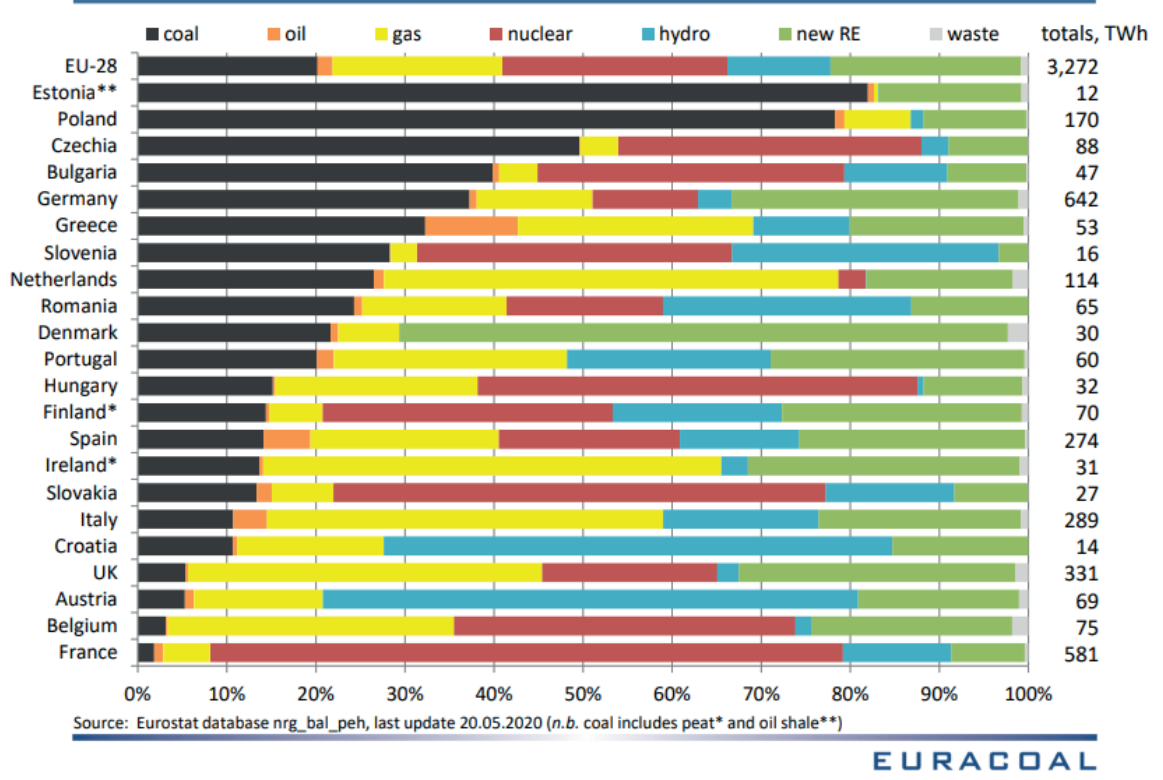

Figure 1 The share of individual sources in electricity production in European countries [28]

Table 1 Comparison of the findings for research measurements to the WLTP procedure recommendations [31]

\begin{tabular}{lcc}
\hline & WLTP Procedure Values & Values adopted for the test \\
\hline Distance covered during the test $[\mathrm{km}]$ & 23.25 & 100 \\
Average speed $[\mathrm{km} / \mathrm{h}]$ & 4.5 & $40-50$ \\
Maximum speed $[\mathrm{km} / \mathrm{h}]$ & 131 & 90 \\
Ambient temperature $\left[{ }^{\circ} \mathrm{C}\right]$ & 23 & $1-3$ \\
\hline
\end{tabular}

the least consuming fossil fuels for energy production countries was Sweden, where the share of fossil fuels in 2020 was only $2 \%$ [27].

A hybrid vehicle is not entirely dependent on electricity, unlike an electric vehicle, as it can only be powered by an internal combustion engine. Charging batteries in electric vehicles can be done at home, thanks to private chargers, or at a charging station. Depending on the current used, two types of chargers can be distinguished. The AC chargers using alternating current with a voltage of $230-400 \mathrm{~V}$. Their charging power ranges from 2 to $22 \mathrm{~kW}$. The DC chargers operate on direct current with a voltage of $400-800 \mathrm{~V}$ and charge with a power of $50-350 \mathrm{~kW}[29]$. At the end of 2020 , there were 1,364 charging stations in Poland. $452(33 \%)$ are fast charging stations and $912(67 \%)$ are the AC stations using alternating current [30].

\section{Materials and methods}

The question of the ecological, economic and utility aspect of a vehicle is the future reference. In each of the above aspects, fuel or energy consumption contributes to improving their efficiency. The selection is made according to the type fuel used to run a vehicle's engine. A study was made of the average fuel/energy consumption of electric, hybrid and spark and selfignition vehicles over a test distance of $100 \mathrm{~km}$. The tests were carried out on four labels, i.e. electric, hybrid, spark ignition and self-ignition. Three vehicles were selected for each group and three measurements were made on them to differentiate between the average fuel or energy consumption.

In order to obtain meaningful results, it was determined that the objectives had been previously met for the WLTP procedures. The Worldwide Harmonized Light Vehicle Test Procedure (WLTP) is a test for testing new production vehicles for emissions and fuel consumption. It is important that this procedure is technical, takes into account large speed fluctuations and allows obtaining more accurate data close to the real ones [31]. The test is conducted according to the established principles of methods based on the WLTP procedures to ensure the results of the consumption analysis and their provider of assurance and reliability services.

\section{Assumptions for measurements}

Table 1 presents selected values of the WLTP procedure and the values adopted for the tests. The selection of values for measurements was made taking 


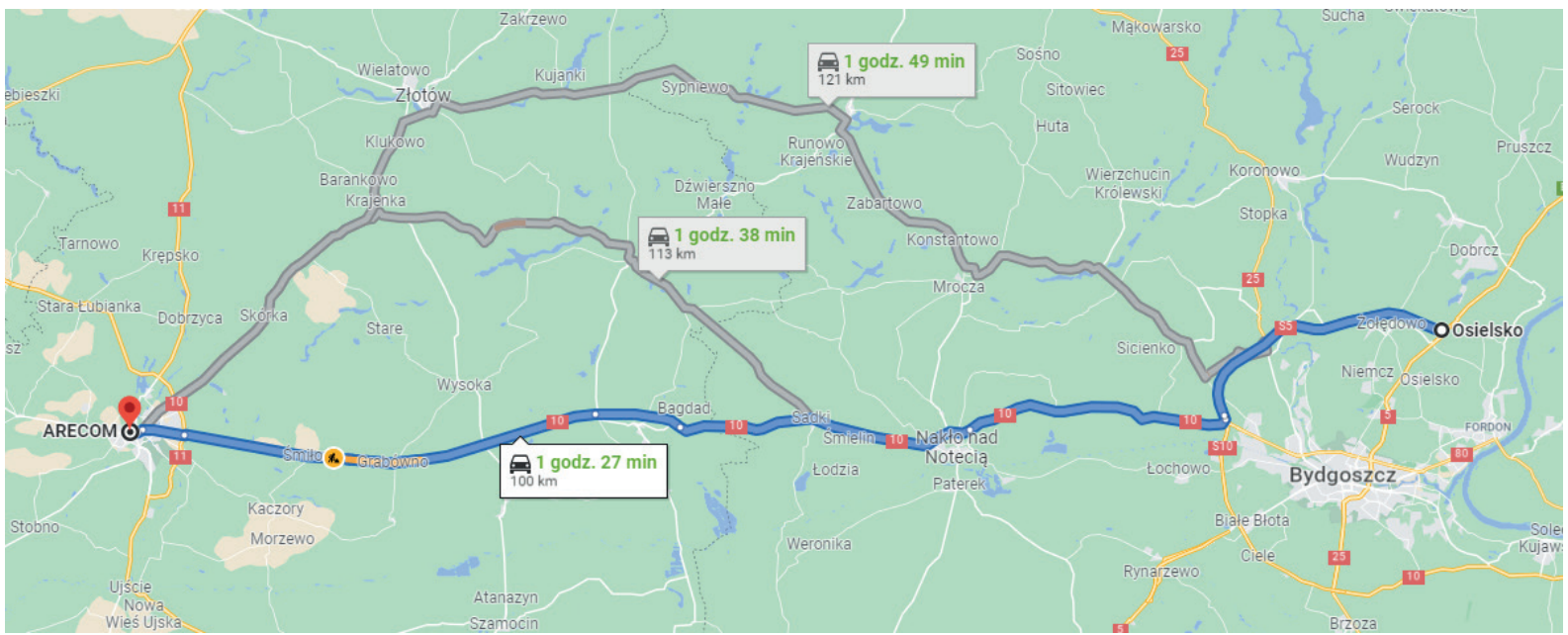

Figure 2 Test section [32]

into account the possibilities and availability of research resources.

The tests were carried out in early winter conditions, which excluded the possibility of setting the temperature higher than $4^{\circ} \mathrm{C}$, the speed could not exceed a certain maximum value for legal reasons, because the vehicles travelled in public traffic, where the maximum permissible speed outside the city is $90 \mathrm{~km} / \mathrm{h}$.

\section{The course of measurements}

Before starting the measurements, the vehicle was refuelled (for conventional and hybrid vehicles) or charged (for electric and hybrid vehicles) to full and then waited for about $3 \mathrm{~h}$ without starting. The engine was then idled for 2 min before starting. After the designated time, the measurement was started. The car was driven on a flat road along the same 100 $\mathrm{km}$ route (from Pila to Osiek, Figure 2) in the city and outside the city by one driver. In order to ensure equal traffic volume conditions between the measurements, the measurements were made on Sunday mornings with low traffic intensity. The interior heating and mandatory lighting were turned on in the vehicle; other additional vehicle equipment was turned off. Fuel consumption was measured using a GPI-EX electronic fuel flow meter and the amount of energy consumed was measured based on the state of charge of the batteries. Weather conditions during the measurements were similar for all the vehicles: no precipitation, no wind, ambient temperature $1-3{ }^{\circ} \mathrm{C}$. There were no traffic disturbances during the measurements and there were no repair or clean-up activities on the road that could affect the speed profile. The same test section was driven 3 times and the arithmetic mean of the fuel/ energy consumed was drawn. In the next step the costs for the above difference were determined based on the prevailing fuel prices $(\mathrm{PB} 95=1.18 € / \mathrm{l} ; \mathrm{ON}=1.16 € / \mathrm{l})$ and energy $0.16 € / \mathrm{kWh}$ )

\section{Research object}

The choice of a vehicle for technical and operational analysis is a multi-element decision, based on an analysis of the market of vehicles available in Poland and Europe and availability. Taking into account the above factors, certain criteria can be defined in order to perform the correct and reliable tests. The selection of vehicles for technical and operational analysis was made using the following criteria:

- Vehicle manufactured after 2015 - the vehicle selection has been limited to cars from the 20162020 period, as it limits the measurement error caused by vehicle wear, which may have hidden defects and unfitness.

- The vehicle is technically fully operational - the vehicle should have up-to-date technical inspections so that during the measurement activities it does not endanger the life and health of the examiner and the test results are not disturbed by unexpected damage to the vehicle.

Tables 2 and 3 show the technical and operational data of the vehicles accepted for testing.

\section{Results}

\subsection{Analysis of the test results of selected electric vehicles}

Electric vehicles were analyzed for the first time. As it results from the measurements (Table 4) and the calculation of the average energy consumption based on three measurements, the energy consumption declared by the manufacturer in all the analyzed vehicles differs from the actual state. The data discrepancy in this case could have been influenced by the average vehicle speed. According to the analysis of the results, the average energy consumption is higher than declared by the manufacturer, 6-16\% depending on the vehicle, which 
Table 2 Ecological, economic and utility parameters of electric and hybrid vehicles [33]

\begin{tabular}{|c|c|c|c|c|c|c|}
\hline \multirow[b]{2}{*}{ Vehicle Name } & \multicolumn{3}{|c|}{ Electric Vehicles } & \multicolumn{3}{|c|}{ Hybrid Vehicles } \\
\hline & Nissan Leaf II & Bmw i3 & Kia e-Niro & $\begin{array}{c}\text { Skoda Superb } \\
\text { IV }\end{array}$ & Bmw X5 45e & $\begin{array}{l}\text { Volkswagen } \\
\text { Passat GTE }\end{array}$ \\
\hline \multicolumn{7}{|c|}{ Basic Parameters } \\
\hline $\begin{array}{l}\text { Maximum } \\
\text { Power }\end{array}$ & $110 \mathrm{~kW}$ & $125 \mathrm{~kW}$ & $100 \mathrm{~kW}$ & $160 \mathrm{~kW}$ & $290 \mathrm{~kW}$ & $160 \mathrm{~kW}$ \\
\hline Range & $270 \mathrm{~km}$ & $297 \mathrm{~km}$ & $289 \mathrm{~km}$ & $61.9 \mathrm{~km}$ & $77 \mathrm{~km}$ & $60 \mathrm{~km}$ \\
\hline Co2 Emissions & $0 \mathrm{~g} / \mathrm{km}$ & $0 \mathrm{~g} / \mathrm{km}$ & $0 \mathrm{~g} / \mathrm{km}$ & $38 \mathrm{~g} / \mathrm{km}$ & $36 \mathrm{~g} / \mathrm{km}$ & $27 \mathrm{~g} / \mathrm{km}$ \\
\hline $\begin{array}{c}\text { Year of } \\
\text { Production }\end{array}$ & 2017 & 2018 & 2020 & 2019 & 2019 & 2019 \\
\hline Mileage & $39906 \mathrm{~km}$ & $40134 \mathrm{~km}$ & $33145 \mathrm{~km}$ & $53402 \mathrm{~km}$ & $27025 \mathrm{~km}$ & $24518 \mathrm{~km}$ \\
\hline \multicolumn{7}{|c|}{ Electric Engine } \\
\hline Engine type & Synchronous & Synchronous & Synchronous & Synchronous & Synchronous & Synchronous \\
\hline $\begin{array}{l}\text { Maximum } \\
\text { power }\end{array}$ & $110 \mathrm{~kW}$ & $125 \mathrm{~kW}$ & $100 \mathrm{~kW}$ & $85 \mathrm{~kW}$ & $83 \mathrm{~kW}$ & $85 \mathrm{~kW}$ \\
\hline $\begin{array}{l}\text { Maximum } \\
\text { torque }\end{array}$ & $320 \mathrm{Nm}$ & $250 \mathrm{Nm}$ & $395 \mathrm{Nm}$ & $330 \mathrm{Nm}$ & $265 \mathrm{Nm}$ & $330 \mathrm{Nm}$ \\
\hline \multicolumn{7}{|c|}{ Charge Time - According to the Manufacturer } \\
\hline $\begin{array}{c}\text { Household } \\
\text { socket } 2.3 \mathrm{~kW}\end{array}$ & $21 \mathrm{~h}$ & $18 \mathrm{~h}$ & $18 \mathrm{~h}$ & $5 \mathrm{~h}$ & $11: 48 \mathrm{~h}$ & $5 \mathrm{~h}$ \\
\hline $11 \mathrm{~kW}$ wallbox & $7: 30 . \mathrm{h}$ & $4 \mathrm{~h}$ & $7 \mathrm{~h}$ & $3: 30 \mathrm{~h}$ & $6: 48 \mathrm{~h}$ & $3: 30 \mathrm{~h}$ \\
\hline $\begin{array}{c}\text { DC } 50 \mathrm{~kW} \\
\text { charging station }\end{array}$ & $1 \mathrm{~h}$ & $0: 45 \mathrm{~h}$ & $0: 57 \mathrm{~h}$ & No DC jack & No DC jack & No DC jack \\
\hline \multicolumn{7}{|c|}{ Battery } \\
\hline Capacity & $40 \mathrm{kWh}$ & $42.2 \mathrm{kWh}$ & $42 \mathrm{kWh}$ & $13 \mathrm{kWh}$ & $24 \mathrm{kWh}$ & $13 \mathrm{kWh}$ \\
\hline \multicolumn{7}{|c|}{ Engine } \\
\hline Engine type & not applicable & not applicable & not applicable & Spark ignition & Spark ignition & Spark ignition \\
\hline $\begin{array}{l}\text { Maximum } \\
\text { power }\end{array}$ & not applicable & not applicable & not applicable & $110 \mathrm{~kW}$ & $210 \mathrm{~kW}$ & $115 \mathrm{~kW}$ \\
\hline $\begin{array}{l}\text { Maximum } \\
\text { torque }\end{array}$ & not applicable & not applicable & not applicable & $250 \mathrm{Nm}$ & $450 \mathrm{Nm}$ & $250 \mathrm{Nm}$ \\
\hline \multicolumn{7}{|c|}{ Performance } \\
\hline Maximum speed & $144 \mathrm{~km} / \mathrm{h}$ & $150 \mathrm{~km} / \mathrm{h}$ & $155 \mathrm{~km} / \mathrm{h}$ & $224 \mathrm{~km} / \mathrm{h}$ & $235 \mathrm{~km} / \mathrm{h}$ & $222 \mathrm{~km} / \mathrm{h}$ \\
\hline $\begin{array}{l}\text { Acceleration } 0 \text { - } \\
100 \mathrm{~km} / \mathrm{h}\end{array}$ & $7.9 \mathrm{~s}$ & $7.3 \mathrm{~s}$ & $9.8 \mathrm{~s}$ & $7.7 \mathrm{~s}$ & $5.6 \mathrm{~s}$ & $7.4 \mathrm{~s}$ \\
\hline $\begin{array}{l}\text { Mixed cycle } \\
\text { energy } \\
\text { consumption }\end{array}$ & $\begin{array}{c}16.9 \mathrm{kWh} / 100 \\
\mathrm{~km}\end{array}$ & $\begin{array}{c}15.8 \mathrm{kWh} / 100 \\
\mathrm{~km}\end{array}$ & $\begin{array}{c}15.3 \mathrm{kWh} / 100 \\
\mathrm{~km}\end{array}$ & $\begin{array}{c}17.3 \mathrm{kWh} / 100 \\
\mathrm{~km}\end{array}$ & $\begin{array}{c}25.7 \mathrm{kWh} / 100 \\
\mathrm{~km}\end{array}$ & $15.7 \mathrm{kWh} / 100 \mathrm{~km}$ \\
\hline $\begin{array}{c}\text { Fuel } \\
\text { consumption }\end{array}$ & - & - & - & $1.9 \mathrm{l} / 100 \mathrm{~km}$ & $1.6 \mathrm{l} / 100 \mathrm{~km}$ & $1.2 \mathrm{l} / 100 \mathrm{~km}$ \\
\hline Range & $270 \mathrm{~km}$ & $297 \mathrm{~km}$ & $289 \mathrm{~km}$ & $61.9 \mathrm{~km}$ & $77 \mathrm{~km}$ & $60 \mathrm{~km}$ \\
\hline \multicolumn{7}{|c|}{ Dimensions } \\
\hline Length & $4490 \mathrm{~mm}$ & $4011 \mathrm{~mm}$ & $4375 \mathrm{~mm}$ & $4869 \mathrm{~mm}$ & $4922 \mathrm{~mm}$ & $4775 \mathrm{~mm}$ \\
\hline Width & $1788 \mathrm{~mm}$ & $1775 \mathrm{~mm}$ & $1805 \mathrm{~mm}$ & $1864 \mathrm{~mm}$ & $2004 \mathrm{~mm}$ & $1832 \mathrm{~mm}$ \\
\hline Height & $1530 \mathrm{~mm}$ & $1577 \mathrm{~mm}$ & $1560 \mathrm{~mm}$ & $1468 \mathrm{~mm}$ & $1745 \mathrm{~mm}$ & $1483 \mathrm{~mm}$ \\
\hline & & & & & & $2783 \mathrm{~mm}$ \\
\hline Wheelbase & $2700 \mathrm{~mm}$ & $2570 \mathrm{~mm}$ & $2700 \mathrm{~mm}$ & $2841 \mathrm{~mm}$ & $2975 \mathrm{~mm}$ & \\
\hline $\begin{array}{l}\text { Front / rear } \\
\text { track }\end{array}$ & $1540 / 1555 \mathrm{~mm}$ & $1571 / 1576 \mathrm{~mm}$ & $1576 / 1585 \mathrm{~mm}$ & $1584 / 1570 \mathrm{~mm}$ & $1656 / 1686 \mathrm{~mm}$ & $1578 / 1586 \mathrm{~mm}$ \\
\hline $\begin{array}{c}\text { Luggage } \\
\text { compartment } \\
\text { capacity }\end{array}$ & 3851 & 2601 & 4511 & 4851 & 5001 & 4021 \\
\hline \multicolumn{7}{|c|}{ Mass } \\
\hline $\begin{array}{l}\text { Minimum empty } \\
\text { weight }\end{array}$ & $1545 \mathrm{~kg}$ & $1345 \mathrm{~kg}$ & $1592 \mathrm{~kg}$ & $1730 \mathrm{~kg}$ & $2510 \mathrm{~kg}$ & $1729 \mathrm{~kg}$ \\
\hline $\begin{array}{l}\text { Maximum total } \\
\text { weight }\end{array}$ & 1995 kg & $1710 \mathrm{~kg}$ & $2080 \mathrm{~kg}$ & $2251 \mathrm{~kg}$ & $3150 \mathrm{~kg}$ & $2230 \mathrm{~kg}$ \\
\hline
\end{tabular}


Table 3 Ecological, economic and operational parameters of vehicles with spark-ignition and self-ignition engines [34]

\begin{tabular}{|c|c|c|c|c|c|c|}
\hline & \multicolumn{3}{|c|}{ Vehicles with Spark-Ignition Engines } & \multicolumn{3}{|c|}{ Compression-Ignition Vehicles } \\
\hline Vehicle Name & BMW X4 & Skoda Rapid II & Opel Corsa E & BMW 5 F10 & Audi A4 B9 & $\begin{array}{l}\text { Renault Megane } \\
\text { IV GrandTour }\end{array}$ \\
\hline \multicolumn{7}{|c|}{ Basic Parameters } \\
\hline Maximum power [kW] & 135 & 80 & 55 & 140 & 110 & 66 \\
\hline Range & $890 \mathrm{~km}$ & $1196 \mathrm{~km}$ & $776 \mathrm{~km}$ & $1400 \mathrm{~km}$ & $1381 \mathrm{~km}$ & $1270 \mathrm{~km}$ \\
\hline $\mathrm{Co}_{2}$ emissions & $168 \mathrm{~g} / \mathrm{km}$ & $106 \mathrm{~g} / \mathrm{km}$ & $132 \mathrm{~g} / \mathrm{km}$ & $132 \mathrm{~g} / \mathrm{km}$ & $111 \mathrm{~g} / \mathrm{km}$ & $195 \mathrm{~g} / \mathrm{km}$ \\
\hline Year of production & 2019 & 2018 & 2017 & 2016 & 2017 & 2016 \\
\hline Mileage & $69952 \mathrm{~km}$ & $65478 \mathrm{~km}$ & $757875 \mathrm{~km}$ & $61578 \mathrm{~km}$ & $72547 \mathrm{~km}$ & $51265 \mathrm{~km}$ \\
\hline \multicolumn{7}{|c|}{ Engine } \\
\hline Engine type & Spark ignition & Spark ignition & Spark ignition & Self-ignition & Self-ignition & Self-ignition \\
\hline Maximum power & $135 \mathrm{~kW}$ & $80 \mathrm{~kW}$ & $55 \mathrm{~kW}$ & $140 \mathrm{~kW}$ & $110 \mathrm{~kW}$ & $66 \mathrm{~kW}$ \\
\hline Maximum torque & $290 \mathrm{Nm}$ & $200 \mathrm{Nm}$ & $130 \mathrm{Nm}$ & $400 \mathrm{Nm}$ & $340 \mathrm{Nm}$ & $220 \mathrm{Nm}$ \\
\hline \multicolumn{7}{|c|}{ Performance } \\
\hline Maximum speed & $215 \mathrm{~km} / \mathrm{h}$ & $200 \mathrm{~km} / \mathrm{h}$ & $167 \mathrm{~km} / \mathrm{h}$ & $229 \mathrm{~km} / \mathrm{h}$ & $221 \mathrm{~km} / \mathrm{h}$ & $174 \mathrm{~km} / \mathrm{h}$ \\
\hline $\begin{array}{c}\text { Acceleration } 0-100 \\
\mathrm{~km} / \mathrm{h}\end{array}$ & $8.3 \mathrm{~s}$ & $9.8 \mathrm{~s}$ & $14 \mathrm{~s}$ & $8.1 \mathrm{~s}$ & $8.9 \mathrm{~s}$ & $14.1 \mathrm{~s}$ \\
\hline Fuel consumption & $7.3 \mathrm{l} / 100 \mathrm{~km}$ & $4.6 \mathrm{l} / 100 \mathrm{~km}$ & $5.8 \mathrm{l} / 100 \mathrm{~km}$ & $5.0 \mathrm{l} / 100 \mathrm{~km}$ & $4.2 \mathrm{l} / 100 \mathrm{~km}$ & $3.7 \mathrm{l} / 100 \mathrm{~km}$ \\
\hline \multicolumn{7}{|c|}{ Dimensions } \\
\hline Length & $4752 \mathrm{~mm}$ & $4483 \mathrm{~mm}$ & $4021 \mathrm{~mm}$ & $4907 \mathrm{~mm}$ & $4726 \mathrm{~mm}$ & $4626 \mathrm{~mm}$ \\
\hline Width & $1918 \mathrm{~mm}$ & $1706 \mathrm{~mm}$ & $1746 \mathrm{~mm}$ & $1860 \mathrm{~mm}$ & $1842 \mathrm{~mm}$ & $1814 \mathrm{~mm}$ \\
\hline Height & $1621 \mathrm{~mm}$ & $1461 \mathrm{~mm}$ & $1481 \mathrm{~mm}$ & $1462 \mathrm{~mm}$ & $1427 \mathrm{~mm}$ & $1457 \mathrm{~mm}$ \\
\hline Wheelbase & $2864 \mathrm{~mm}$ & $2602 \mathrm{~mm}$ & $2510 \mathrm{~mm}$ & $2968 \mathrm{~mm}$ & $2820 \mathrm{~mm}$ & $2717 \mathrm{~mm}$ \\
\hline Front / rear track & $1620 / 1666 \mathrm{~mm}$ & $1463 / 1500 \mathrm{~mm}$ & $1472 / 1472 \mathrm{~mm}$ & $1600 / 1626 \mathrm{~mm}$ & $1572 / 1555 \mathrm{~mm}$ & $1591 / 1586 \mathrm{~mm}$ \\
\hline $\begin{array}{l}\text { Luggage compartment } \\
\text { capacity }\end{array}$ & 5251 & 5501 & 2851 & 5601 & 4801 & 5801 \\
\hline \multicolumn{7}{|c|}{ Mass } \\
\hline $\begin{array}{l}\text { Minimum empty } \\
\text { weight }\end{array}$ & $1795 \mathrm{~kg}$ & $1170 \mathrm{~kg}$ & $1081 \mathrm{~kg}$ & $1800 \mathrm{~kg}$ & $1505 \mathrm{~kg}$ & $1312 \mathrm{~kg}$ \\
\hline $\begin{array}{l}\text { Maximum total } \\
\text { weight }\end{array}$ & $2400 \mathrm{~kg}$ & $1630 \mathrm{~kg}$ & 1595 & $2375 \mathrm{~kg}$ & $2030 \mathrm{~kg}$ & 1911 \\
\hline
\end{tabular}

is $€ 0.20-040$ respectively. Carrying out tests in winter, at a temperature of $1-3{ }^{\circ} \mathrm{C}$, affects the battery and its discharge rate.

\subsection{Analysis of the test results of selected hybrid vehicles}

Hybrid vehicles are another of the analyzed vehicles. Based on the analysis of the test results presented in Table 5, it was found that the change in values of individual results, as in the case of electric vehicles, could have been influenced by the average speed during the measurements. The analysis showed significant differences between the fuel and energy consumption stated by the manufacturer and the test result, exceeding $189 \%$. This is due to the fact that the tests were carried out in the mixed driving mode, so the tested vehicle was forced to frequent momentary changes in speed, which forced the internal combustion engine to start and thus the use of conventional fuel. As the measurements show, this resulted in an increase in fuel consumption. This result is highly unsatisfactory due to the significant differences in fuel consumption. Taking into account the costs of electricity consumption, one will cover 100 $\mathrm{km}$ with the cheapest Volkswagen Passat GTE and Skoda Superb IV and the most expensive BMW X5 45e. The difference between the energy consumption value declared by the manufacturer and the measured value is small, about $20 \%$.

\subsection{Analysis of the test results of selected vehicles with a spark ignition engine}

A spark-ignition car was analyzed successively. After analyzing the data in Table 6, the average fuel consumption may amount to 25-30 \% - which corresponds to $1.6-2.1 €$, higher than declared by the manufacturer. The exception is the Opel Corsa, where the manufacturer's results are consistent with the data obtained during the measurements. A slight change in the average speed has little effect on the amount of fuel consumed, with the exception of the Skoda Rapid. The reason for the increase in fuel consumption was the low temperature during the tests. 
Table 4 Electric vehicle test results [35-37]

\begin{tabular}{|c|c|c|c|c|c|c|c|c|c|}
\hline \multirow{3}{*}{$\begin{array}{l}\text { Car data / model } \\
\text { Data }\end{array}$} & \multicolumn{3}{|c|}{ Nissan Leaf 2} & \multicolumn{3}{|c|}{ BMW i3 } & \multicolumn{3}{|c|}{ Kia e-Niro } \\
\hline & Test & Test & Test & Test & Test & Test & Test & Test & Test \\
\hline & 1 & 2 & 3 & 1 & 2 & 3 & 1 & 2 & 3 \\
\hline Distance [km] & 100 & 100 & 100 & 100 & 100 & 100 & 100 & 100 & 100 \\
\hline Average speed [km/h] & 43 & 46 & 44 & 45 & 41 & 43 & 44 & 46 & 43 \\
\hline Temperature $\left[{ }^{\circ} \mathrm{C}\right]$ & 1 & 2 & 1 & 2 & 3 & 2 & 3 & 3 & 3 \\
\hline \multirow{2}{*}{ Energy consumption per $100 \mathrm{~km}[\mathrm{kWh}] /$ value $[€]$} & $17.7 /$ & $18.7 /$ & \multirow{2}{*}{$17.9 / 2.86$} & $17.8 /$ & $16 /$ & $16.5 /$ & $17.6 /$ & $18.1 /$ & $17.6 /$ \\
\hline & 2.83 & 2.99 & & 2.85 & 2.56 & 2.64 & 2.82 & 2.90 & 2.82 \\
\hline Average energy consumption per $100 \mathrm{~km}[\mathrm{kWh}] /$ value $[€]$ & \multicolumn{3}{|c|}{$18.1 / 2.89$} & \multicolumn{3}{|c|}{$16.8 / 2.68$} & \multicolumn{3}{|c|}{$17.8 / 2.85$} \\
\hline Manufacturer declared consumption $[\mathrm{kWh}] /$ value $[€]$ & \multicolumn{3}{|c|}{$16.9 / 2.70$} & \multicolumn{3}{|c|}{$15.8 / 2.53$} & \multicolumn{3}{|c|}{$15.3 / 2.45$} \\
\hline Difference in energy consumption [\%] / value [€] & \multicolumn{3}{|c|}{$7.1 / 0.19$} & \multicolumn{3}{|c|}{$6.3 / 0.15$} & \multicolumn{3}{|c|}{$16.3 / 0.40$} \\
\hline Average $[\%]$ / value $[€]$ & \multicolumn{9}{|c|}{$9.9 / 0.25$} \\
\hline
\end{tabular}

Table 5 Test results for hybrid vehicles [37-39]

\begin{tabular}{|c|c|c|c|c|c|c|c|c|c|}
\hline \multirow{3}{*}{$\begin{array}{l}\text { Car data / model } \\
\text { Data }\end{array}$} & \multicolumn{3}{|c|}{ BMW X5 45e } & \multicolumn{3}{|c|}{ Skoda Superb IV } & \multicolumn{3}{|c|}{ Volkswagen Passat GTE } \\
\hline & Test & Test & Test & Test & Test & Test & Test & Test & Test \\
\hline & 1 & 2 & 3 & 1 & 2 & 3 & 1 & 2 & 3 \\
\hline Distance [km] & 100 & 100 & 100 & 100 & 100 & 100 & 100.1 & 100 & 100.1 \\
\hline Average speed $[\mathrm{km} / \mathrm{h}]$ & 47 & 43 & 44 & 45 & 46 & 45 & 49 & 47 & 48 \\
\hline Temperature $\left[{ }^{\circ} \mathrm{C}\right]$ & 3 & 3 & 3 & 2 & 2 & 2 & 2 & 2 & 1 \\
\hline $\begin{array}{l}\text { Fuel consumption per } 100 \mathrm{~km}[\mathrm{l}] \\
\text { / value }[€]\end{array}$ & $7.5 / 8.85$ & $7.2 / 8.50$ & $7.4 / 8.73$ & $5.5 / 6.49$ & $5.6 / 6.61$ & $5.5 / 6.49$ & $6.5 / 7.67$ & $6.5 / 7.67$ & $6.5 / 7.67$ \\
\hline $\begin{array}{l}\text { Average fuel consumption per } \\
100 \mathrm{~km}[1] / \text { value }[€]\end{array}$ & & $7.4 / 8.69$ & & & $5.5 / 6.53$ & & & $6.5 / 7.67$ & \\
\hline $\begin{array}{l}\text { Fuel consumption declared by } \\
\text { the manufacturer [l] / value [€] }\end{array}$ & & $2.1 / 2.48$ & & & $1.9 / 2.24$ & & & $2.1 / 2.48$ & \\
\hline $\begin{array}{l}\text { Fuel consumption difference [\%] } \\
\text { / value }[€]\end{array}$ & & $252.4 / 6.21$ & & & $189.5 / 4.29$ & & & 209.5/ 5.19 & \\
\hline Average [\%] / value $[€]$ & & & & & $234.1 / 5.23$ & & & & \\
\hline $\begin{array}{l}\text { Energy consumption per } 100 \mathrm{~km} \\
{[\mathrm{kWh}] / \text { value }[€]}\end{array}$ & $\begin{array}{c}36.92 / \\
5.91\end{array}$ & $\begin{array}{l}35.14 / \\
5.62\end{array}$ & $\begin{array}{l}37.14 / \\
5.94\end{array}$ & $\begin{array}{c}26.00 / \\
4.16\end{array}$ & $\begin{array}{c}25.15 / \\
4.02\end{array}$ & $\begin{array}{l}22.81 / \\
3.65\end{array}$ & $\begin{array}{c}21.67 / \\
3.47\end{array}$ & $\begin{array}{l}22.80 / \\
3.65\end{array}$ & $\begin{array}{l}24.07 / \\
3.85\end{array}$ \\
\hline $\begin{array}{l}\text { Average energy consumption per } \\
100 \mathrm{~km}[\mathrm{kWh}] / \text { value }[€]\end{array}$ & & $36.40 / 5.82$ & & & $24.65 / 3.94$ & & & $22.85 / 3.66$ & \\
\hline $\begin{array}{l}\text { Manufacturer declared } \\
\text { consumption }[\mathrm{kWh}] / \text { value }[€]\end{array}$ & & $29.10 / 4.66$ & & & $21.31 / 3.41$ & & & $20.31 / 3.23$ & \\
\hline $\begin{array}{l}\text { Difference in energy } \\
\text { consumption }[\%] \text { / value }[€]\end{array}$ & & $25.1 / 1.16$ & & & $20.4 / 0.53$ & & & $12.51 / 0.43$ & \\
\hline Average [\%] / value [€] & & & & & $19.34 / 0.71$ & & & & \\
\hline
\end{tabular}

Table 6 Test results for vehicles with a positive-ignition engine [37-38, 40]

\begin{tabular}{|c|c|c|c|c|c|c|c|c|c|}
\hline \multirow{3}{*}{$\begin{array}{l}\text { Car data / model } \\
\text { Data }\end{array}$} & \multicolumn{3}{|c|}{ BMW X4 } & \multicolumn{3}{|c|}{ Skoda Rapid II } & \multicolumn{3}{|c|}{ Opel Corsa E } \\
\hline & Test & Test & Test & Test & Test & Test & Test & Test & Test \\
\hline & 1 & 2 & 3 & 1 & 2 & 3 & 1 & 2 & 3 \\
\hline Distance $[\mathrm{km}]$ & 100 & 100 & 100 & 100 & 100 & 100 & 100 & 100 & 100 \\
\hline Average speed $[\mathrm{km} / \mathrm{h}]$ & 46 & 48 & 46 & 42 & 45 & 43 & 47 & 47 & 47 \\
\hline Temperature $\left[{ }^{\circ} \mathrm{C}\right]$ & 1 & 1 & 1 & 2 & 2 & 2 & 2 & 3 & 2 \\
\hline 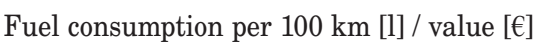 & \multicolumn{9}{|c|}{ 9.1/ 10.749.1/ 10.749.1/ 10.745.9/6.966.4/ 7.555.5/6.495.7/6.735.8/6.84 5.8/6.84 } \\
\hline $\begin{array}{l}\text { Average fuel consumption per } 100 \mathrm{~km}[1] / \\
\text { value }[€]\end{array}$ & \multicolumn{3}{|c|}{$9.1 / 10.74$} & \multicolumn{3}{|c|}{$5.9 / 7.00$} & \multicolumn{3}{|c|}{$5.8 / 6.84$} \\
\hline $\begin{array}{l}\text { Fuel consumption declared by the } \\
\text { manufacturer }[1] / \text { value }[€]\end{array}$ & \multicolumn{3}{|c|}{$7.3 / 8.61$} & \multicolumn{3}{|c|}{$4.6 / 5.43$} & \multicolumn{3}{|c|}{$5.8 / 6.84$} \\
\hline Fuel consumption difference $[\%]$ / value $[€]$ & \multicolumn{3}{|c|}{$24.7 / 2.1$} & \multicolumn{3}{|c|}{$28.3 / 1.57$} & \multicolumn{3}{|c|}{$0 / 0$} \\
\hline Average $[\%] /$ value $[€]$ & \multicolumn{9}{|c|}{$17.7 / 1.22$} \\
\hline
\end{tabular}


Table 7 The results of tests of vehicles with a compression-ignition engine [37, 41-42]

\begin{tabular}{|c|c|c|c|c|c|c|c|c|c|}
\hline \multirow{3}{*}{$\begin{array}{l}\text { Car data / model } \\
\text { Data }\end{array}$} & \multicolumn{3}{|c|}{ BMW 5 F10 } & \multicolumn{3}{|c|}{ Audi A4 B9 } & \multicolumn{3}{|c|}{$\begin{array}{c}\text { Renault Megane IV } \\
\text { GrandTour }\end{array}$} \\
\hline & Test & Test & Test & Test & Test & Test & Test & Test & Test \\
\hline & 1 & 2 & 3 & 1 & 2 & 3 & 1 & 2 & 3 \\
\hline Distance $[\mathrm{km}]$ & 100 & 100 & 100 & 100 & 100 & 100 & 100 & 100 & 100 \\
\hline Average speed $[\mathrm{km} / \mathrm{h}]$ & 46 & 45 & 46 & 49 & 47 & 49 & 44 & 44 & 42 \\
\hline Temperature $\left[{ }^{\circ} \mathrm{C}\right]$ & 4 & 4 & 4 & 2 & 2 & 2 & 3 & 3 & 3 \\
\hline Fuel consumption per $100 \mathrm{~km} \mathrm{[l]} \mathrm{/} \mathrm{value} \mathrm{[€]}$ & $\begin{array}{l}7.3 / \\
8.47\end{array}$ & $\begin{array}{l}7.3 / \\
8.47\end{array}$ & $\begin{array}{l}7.3 / \\
8.47\end{array}$ & $\begin{array}{l}6.3 / \\
7.31\end{array}$ & $\begin{array}{l}6.3 / \\
7.31\end{array}$ & $\begin{array}{l}6.4 / \\
7.42\end{array}$ & $\begin{array}{l}6.2 / \\
7.19\end{array}$ & $\begin{array}{l}6.2 / \\
7.19\end{array}$ & $\begin{array}{l}6.1 / \\
7.08\end{array}$ \\
\hline $\begin{array}{l}\text { Average fuel consumption per } 100 \mathrm{~km}[1] / \\
\text { value }[€]\end{array}$ & \multicolumn{3}{|c|}{$7.3 / 8.47$} & \multicolumn{3}{|c|}{$6.3 / 7.35$} & \multicolumn{3}{|c|}{$6.2 / 7.14$} \\
\hline $\begin{array}{l}\text { Fuel consumption declared by the } \\
\text { manufacturer }[1] \text { / value }[€]\end{array}$ & \multicolumn{3}{|c|}{$5 / 5.80$} & \multicolumn{3}{|c|}{$4.2 / 4.87$} & \multicolumn{3}{|c|}{$3.7 / 4.29$} \\
\hline Fuel consumption difference [\%] / value $[€]$ & \multicolumn{3}{|c|}{$46 / 2.67$} & \multicolumn{3}{|c|}{$50 / 2.48$} & \multicolumn{3}{|c|}{$67.6 / 2.85$} \\
\hline Average $[\%] /$ value $[€]$ & & & & \multicolumn{3}{|c|}{$54.2 / 2.67$} & & & \\
\hline
\end{tabular}

\subsection{Analysis of the test results of selected diesel vehicles}

Self-ignition cars are the last of the studied groups of vehicles. The data in Table 7 shows that during the measurements the vehicles maintained an average speed of $42-49 \mathrm{~km} / \mathrm{h}$ and their fuel consumption was higher than the manufacturer's assurances by 46-65 $\%$. This is due to the conditions under which the test was carried out and also due to the temperature, the fuel demand increased, which in turn increased its consumption. These results are below expectations because the Renault Megane IV GrandTour, despite the low engine power, does not differ much from the other tested diesel vehicles.

\section{Discussion}

In order to accurately illustrate the differences between the actual fuel consumption and declared by the manufacturer, the percentage difference between the obtained results was compared. As shown by the data in the Table 8 , the discrepancies are huge and surprising. Despite the opinion that hybrid vehicles, apart from electric vehicles, are economical, the research results showed that the difference in fuel consumption between the measured value and the value declared by the manufacturer is huge and amounts to $234.1 \%$. The fuel consumption of the diesel vehicles is $54.2 \%$ higher.
Energy consumption in electric vehicles was on average higher by $9.9 \%$, which in the case of low temperatures is a satisfactory result. Taking into account the costs difference between the declared and measured fuel consumption by the tested vehicles, it follows that the smallest difference occurs in the case of electric vehicles and the largest in the case of hybrid vehicles.

\section{Conclusions}

Assessing all the obtained results, it can be concluded that the level of fuel/energy consumption remains at an acceptable level. The differences were largely due to independent factors such as the weather conditions. The air temperature adopted by the manufacturer during the test was $23{ }^{\circ} \mathrm{C}$ and during the tests it did not exceed $4{ }^{\circ} \mathrm{C}$. The air temperature has a great influence on the combustion of the fuel-air mixture. Based on the research, it can be assumed that the differences of 10-20 $\%$ in winter conditions are acceptable and the energy consumption of electric vehicles is also within the limits that allow one to believe that these vehicles are a good replacement for the conventional ones.

One of the important elements that the average car user pays attention to is the fuel consumption of a given vehicle. The conducted research shows that in terms of ecology, hybrid vehicles fulfil their role as a replacement for a combustion vehicle and in terms of fuel consumption and the economic aspect, it can be

Table 8 Average difference in fuel consumption of the tested vehicles

\begin{tabular}{lcc}
\hline Vehicle type & Average percentage difference & Cost [€] \\
\hline Electric vehicles & 9.9 & 0.25 \\
Hybrid vehicles - fuel & 234.1 & 5.94 \\
Hybrid vehicles - energy & 19.34 & 1.22 \\
Vehicles with positive-ignition engine & 17.7 & 2.67 \\
Vehicles with a compression-ignition engine & 54.2 & \\
\hline
\end{tabular}


concluded that dynamic driving and winter conditions are not favorable for these vehicles. The electric vehicle proves in every way that it is the successor to vehicles with internal combustion engines.

\section{References}

[1] KURANC, A., CABAN, J., SARKAN, B., DUDZIAK, A., STOMA, M. Emission of selected exhaust gas components and fuel consumption in different driving cycles. Communications - Scientific Letters of the University of Zilina [online]. 2021, 23(4), p. B265-B277 [accessed 2021-10-19]. ISSN 1335-4205, eISSN 2585-7878. Available from: https://doi.org/10.26552/com.C.2021.4.B265-B277

[2] VERNER, J., SEJKOROVA, M. Comparison of CVS and PEMS measuring devices used for stating CO2 exhaust emissions of light-duty vehicles during WLTP testing procedure. Engineering for Rural Development [online]. 2018, 17, p. 2054-2059. ISSN 1691-5976. Available from: https://doi.org/10.22616/ERDev2018.17.N284

[3] HOOFTMAN, N., MESSAGIE, M., VAN MIERLO, J., COOSEMANS, T. A review of the European passenger car regulations - real driving emissions vs local air quality. Renewable and Sustainable Energy Reviews [online]. 2018, 86, p. 1-21 [accessed 2021-10-19]. ISSN 1364-0321. Available from: https://doi.org/10.1016/j. rser.2018.01.012

[4] CABAN, J., IGNACIUK, P. Technical-economic aspects of CNG gas usage in buses of urban communication. Engineering for Rural Development [online]. 2018, 17, p. 2129-2133. ISSN 1691-5976. Available from: https://doi.org/10.22616/ERDev2018.17.N483

[5] MERKISZ, J., FUC, P., LIJEWSKI, P., PIELECHA, J. Actual emissions from urban buses powered with diesel and gas engines. Transportation Research Procedia [online]. 2016, 14, p. 3070-3078 [accessed 2021-10-19]. ISSN 2352-1465. Available from: https://doi.org/10.1016/j.trpro.2016.05.452

[6] FLEKIEWICZ, M., KUBICA G. The influence of selected gaseous fuels on the combustion process in the $\mathrm{Si}$ engine. Transport Problems [online]. 2017, 12(3), p. 135-146 [accessed 2021-10-19]. eISSN 2300-861X. Available from: https://doi.org/10.20858/tp.2017.12.3.13

[7] KORSAKAS, V., MELAIKA, M., PUKALSKAS, S., STRAVINSKAS, P. Hydrogen addition influence for the efficient and ecological parameters of heavy-duty natural gas Si engine. Procedia Engineering [online]. 2017, 187, p. 395-401 [accessed 2021-10-19]. ISSN 1877-7058. Available from: https://doi.org/10.1016/j.proeng.2017.04.391

[8] SILEGHEM, L., BOSTEELS, D., MAY, J., FAVRE, C., VERHELST, S. Analysis of vehicle emission measurements on the new WLTC, the NEDC and the CADC. Transportation Research Part D: Transport and Environment [online]. 2014, 32, p. 70-85 [accessed 2021-10-19]. ISSN 1361-9209. Available from: https://doi.org/10.1016/j. trd.2014.07.008

[9] DIMARATOS, A., TSOKOLIS, D., FONTARAS, G., TSIAKMAKIS, S., CIUFFO, B., SAMARAS, Z. Comparative evaluation of the effect of various technologies on light-duty vehicle CO2 emissions over NEDC and WLTP. Transportation Research Procedia [online]. 2016, 14, p. 3169-3178 [accessed 2021-10-19]. ISSN 2352-1465. Available from: https://doi.org/10.1016/j.trpro.2016.05.257

[10] MANWARING, L. A. The observer's book of automobiles. Frederick Warne Publishers Ltd., 1961.

[11] FUNDOWICZ, P., RADZIMIERSKI, M., WIECZOREK, M. Construction of motor vehicles (in Polish). Warsaw: WSiP, 2010. ISBN 978-83-02-11720-6.

[12] GABRYELEWICZ, M. Chassis and bodies of motor vehicles 2 (in Polish). Warsaw: WKL, 2012. ISBN 978-83-2062001-1.

[13] GABRYELEWICZ, M., ZAJAC, P. Construction of WKiL motor vehicles (in Polish). Warsaw: WKL, 2019. ISBN 978-83-206-2013-9.

[14] ORZELOWSKI, S. Construction of chassis and car bodies (in Polish). Warsaw: WSiP, 2003. ISBN 978-83-0208785-1.

[15] ORZELOWSKI, S. Repair and maintenance of motor vehicles (in Polish). Warsaw: WSiP, 1996. ISBN 978-83-0213619-1.

[16] ZAJAC, P. Motors of motor vehicles 2 (in Polish). Warsaw: WKL, 2011. ISBN 978-83-206-1783-2.

[17] SCHMIDT, T. Hybrid and electric vehicles in workshop practice (Polish edition). Warsaw: WKL, 2020. ISBN 97883-20-62011-5.

[18] The first hybrid car is 111 years old (in Polish) [online] [accessed 2021-02-17]. Available from: https://motoryzacja. interia.pl/wiadomosci/producenci/news-pierwszy-hybrydowy-samochod-ma-111-lat,nId,1387142

[19] The first Porsche car - Porsche P1 from 1898 (in Polish) [online] [accessed 2021-02-17]. Available from: https://jazda-porsche.pl/pierwszy-samochod-porsche-porsche-p1-z-1898-roku/

[20] STOPKA, O., STOPKOVA, M., LIZBETIN, J., SOVIAR J., CABAN, J. Development trends of electric vehicles in the context of road passenger and freight transport. In: 2020 XII International Science-Technical Conference Automotive Safety: proceedings [online]. IEEE. 2020. p. 1-8. Available from: https://doi.org/10.1109/ AUTOMOTIVESAFETY47494.2020.9293526 
[21] HURTOVA, I., SEJKOROVA, M., VERNER, J., SARKAN, B. Comparison of electricity and fossil fuel consumption in trolleybuses and buses. Engineering for Rural Development [online]. 2018, 17, p. 2079-2084. ISSN 1691-5976. Available from: https://doi.org/10.22616/ERDev2018.17.N342

[22] MALEK, A., CABAN, J., WOJCIECHOWSKI, L. Charging electric cars as a way to increase the use of energy produced from RES. Open Engineering [online]. 2020, 10(1), p. 98-104 [accessed 2021-10-19]. ISSN 2391-5439. Available from: https://doi.org/10.1515/eng-2020-0009

[23] LOSIEWICZ, Z., SENDEK-MATYSIAK, E. The development of electromobility: in terms of the use of electric cars. Autobusy - Technika, Eksploatacja, Systemy Transportowe / Buses - Technology, Operation, Transport Systems [online]. 2018, 19(12), p. 126-128. ISSN 1509-5878, eISSN 2450-7725 Available from: https://doi. org/10.24136/atest.2018.368

[24] MERKISZ, J., PIELECHA, I. Alternative vehicle drives (in Polish). Warsaw: WPP, 2006. ISBN 978-83-71-43260-6.

[25] Electric cars - full offer of the Polish market (in Polish) [online] [accessed 2021-02-17]. Available from: https://www. auto-swiat.pl/wiadomosci/aktualnosci/samochody-elektryczne-pelna-oferta-polskiego-rynku/k9dcx41\#slajd-15

[26] European alternative fuels observatory [online] [accessed 2021-02-17]. Available from: https://www.eafo.eu/

[27] Renault Megane GT: different but or better (in Polish) [online] [accessed 2021-02-17]. Available from: https://www.autocentrum.pl/publikacje/testy-aut-nowych/renault-megane-gt-inna-ale-czy-lepsza/

[28] Electricity generation mix in selected countries [online] [accessed 2021-02-17]. Available from: https://euracoal. eu/info/euracoal-eu-statistics/

[29] Charging electric cars - types of chargers (in Polish) [online] [accessed 2021-02-17]. Available from: https://www.autocentrum.pl/publikacje/porady/ladowanie-samochodow-elektrycznych-rodzaje-ladowarek/

[30] Electromobility meter: 2020 record-breaking on the Polish electric car market (in Polish) [online] [accessed 2021-02-17]. Available from: https://pspa.com.pl/2021/informacja/licznik-elektromobilnosci-rok-2020-rekordowyna-polskim-rynku-samochodow-elektrycznych/

[31] Harmonized procedure for testing vehicles around the world (in Polish) [online] [accessed 2021-02-17]. Available from: https://www.dsautomobiles.pl/swiat-ds/technologie/wltp.html

[32] Maps google [online] [accessed 2021-10-19]. Available from: https://www.google.pl/maps

[33] Electric vehicles catalog (in Polish) [online] [accessed 2021-02-17]. Available from: pspa.com.pl/media/2020/08/ katalog_pojazdow_elektrycznych_2020_S_.pdf

[34] Technical data of vehicles (in Polish) [online] [accessed 2021-02-17]. Available from: www.autocentrum.pl/

[35] Technical data of vehicles - Nissan (in Polish) [online] [accessed 2021-02-17]. Available from: https://www.nissan.pl/

[36] Technical data of vehicles - KIA (in Polish) [online] [accessed 2021-02-17]. Available from: https://www.kia.pl

[37] Technical data of vehicles - BMW (in Polish) [online] [accessed 2021-02-17]. Available from: https://www.bmw.pl/

[38] Technical data of vehicles - Skoda (in Polish) [online] [accessed 2021-02-17]. Available from: https://www.skodaauto.pl/

[39] Technical data of vehicles - Volkswagen (in Polish) [online] [accessed 2021-02-17]. Available from: https://www.volkswagen.pl/

[40] Technical data of vehicles - Opel (in Polish) [online] [accessed 2021-02-17]. Available from: https://www.opel.pl/

[41] Technical data of vehicles - Audi (in Polish) [online] [accessed 2021-02-17]. Available from: https://www.audi.pl/

[42] Technical data of vehicles - Renault (in Polish) [accessed 2021-02-17]. Available from: https://www.renault.pl 